\title{
Latitudinal variation of stochastic properties of the geomagnetic field
}

\author{
J. A. Wanliss ${ }^{1}$, K. Shiokawa ${ }^{2}$, and K. Yumoto ${ }^{3}$ \\ ${ }^{1}$ Presbyterian College, 503 South Broad Street, Clinton, SC 29325, USA \\ ${ }^{2}$ Solar-Terrestrial Environment Laboratory, Nagoya University, Furo-cho, Chikusa-ku, Nagoya, Aichi, 464-8601, Japan \\ ${ }^{3}$ Space Environment Research Center, Kyushu University, 53 6-10-1 Hakozaki, Higashi-ku Fukuoka, 812-8581, Japan
}

Correspondence to: J. A. Wanliss (jawanliss@presby.edu)

Received: 24 August 2012 - Revised: 26 October 2013 - Accepted: 19 January 2014 - Published: 3 March 2014

\begin{abstract}
We explore the stochastic fractal qualities of the geomagnetic field from $210 \mathrm{~mm}$ ground-based magnetometers during quiet and active magnetospheric conditions. We search through $10 \mathrm{yr}$ of these data to find events that qualify as quiet intervals, defined by $\mathrm{Kp} \leq 1$ for 1440 consecutive minutes. Similarly, active intervals require $\mathrm{Kp} \geq 4$ for 1440 consecutive minutes. The total for quiet intervals is $\sim 4.3 \times 10^{6}$ and $2 \times 10^{8} \mathrm{~min}$ for active data points. With this large number of data we characterize changes in the nonlinear statistics of the geomagnetic field via measurements of a fractal scaling. A clear difference in statistical behavior during quiet and active intervals is implied through analysis of the scaling exponents; active intervals generally have larger values of scaling exponents. This suggests that although $210 \mathrm{~mm}$ data appear monofractal on shorter timescales, the scaling changes, with overall variability are more likely described as a multifractional Brownian motion. We also find that low latitudes have scaling exponents that are consistently larger than for high latitudes.
\end{abstract}

\section{Introduction}

The transfer of energy, mass, and momentum from the solar wind into the magnetospheric cavity is far from steady state. Rapid transfers of energy cause the magnetosphere to move from a relatively low-energy state into more "exited" states where energy dissipation occurs suddenly in global dynamical processes known as space storms. They are the most dramatic space weather phenomenon that significantly impact modern technology such as satellites, communication and power transmission systems. Space physicists un- derstand their development and morphology, but discussion is still open regarding detailed causes of space storms.

Energy dissipation in the terrestrial magnetosphere often follows intermittent temporal patterns of high activity with abrupt convection increases, particle bursts, impulsive enhancements of $\mathrm{O}+/ \mathrm{H}+$ density ratio, compressional pulses, intensified fluctuations of field-aligned currents and other bursty processes. These impulsive processes may be separated by periods of quiescence (Korth et al., 2003). Bursty intermittency is evident in both in situ and ground observations (Hori et al., 2005), and are observed under a wide variety of interplanetary conditions (Pulkkinen et al., 2007).

Ground-based geomagnetic indices (Takalo et al., 1999; Wanliss, 2004, 2005; Balasis et al., 2011) and individual magnetometer stations (Vörös, 2000; Wanliss and Reynolds, 2003) provide excellent signals of the fractal nature of space weather energy dissipation. Part of the reason for this is the property of the earth's magnetic field lines to focus and converge when approaching the earth. These field lines extend far into space and since they connect to the earth, nonlinear plasma processes that occur far away map all the way down to the planetary surface. Observation of ground-based magnetometer stations can thus serve as a remote sensing tool of distant magnetospheric features and processes.

Over the years, several indices were developed to monitor geomagnetic activity in a global sense. The most commonly used are the disturbance storm time index (DST), the planetary index (Kp) and the auroral electrojet index (AE, AU and $\mathrm{AL}$ ). These indices provide global information about magnetospheric activity by combining inputs at different locations around the globe. The Kp index, which we utilize in this paper, is calculated with data from 13 selected, subauroral 
magnetometer stations at mid- to high latitudes. Because the stations cover a wide range of latitudes it is a relevant reflection of average global magnetospheric activity.

The $\mathrm{Kp}$ index is obtained as the average value of the disturbance levels in the two horizontal field components, observed at the reference magnetometer stations (Bartels et al., 1939; Bartels, 1963). Calculation of Kp begins by removing the quiet-day variation from the individual magnetograms (cf. Wanliss and Showalter, 2006). The next step finds the range of the horizontal magnetic field disturbances during 3hour time intervals, for eight values each day. At each station the range is converted to a quasi-logarithmic scale index with values from 0 to 9 . The logarithmic scale normalizes the occurrence frequency of different size disturbances. Finally, an averaging procedure convolves the local indices to form the global geomagnetic index $\mathrm{Kp}$.

If we are interested in the local aspects of geomagnetic activity, i.e., to forecast the geomagnetic conditions for power utilities, we need to develop ways to understand the geomagnetic activity in a more localized way. This is especially important since temporal fluctuations of the geomagnetic field and, more importantly, their nonlinear fractal or multifractal statistical fluctuations depend on geographic location and time (Anh et al., 2007; Yu et al., 2010).

Furthermore, the intermittent character of geomagnetic data suggests that changes in the magnetosphere depend not only on solar wind variability, but also on internal magnetospheric activity (Consolini et al., 1996; Consolini and De Michelis, 1998; Dobias and Wanliss, 2009; Wanliss and Uritsky, 2010). The origin of such an intermittent and multifractal character has been interpreted and modeled in terms of dynamical changes and turbulent dynamics (Wanliss et al., 2005; Anh et al., 2007; Yu et al., 2010; Sharma and Veeramani, 2011).

In this paper we extend previous analyses that considered only global statistics to study the differences between quiet and active magnetospheric times (Wanliss, 2004, 2005). Global studies give only average behavior rather than local information. Our goal is to learn about local behavior of the magnetic field, for differing geomagnetic activity. To do so we will characterize changes in the nonlinear statistics of the earth's magnetic field, by means of the fractal scaling (Hurst) exponent, measured from ground-based magnetometer station.

Previous studies by Wanliss and Reynolds (2003) suggested the possibility that the ubiquitous fractal scaling of geomagnetic data may yet have variability on a latitudinal scale. In addition, while the fractal character of geomagnetic data is ubiquitous, there is strong evidence that the fractal character varies with geomagnetic activity (Wanliss and Dobias, 2007).

The present study explores this further, asking the following questions: (1) Does average fractal scaling vary in a predictable way as a function of latitude? and (2) Are magneto- spherically quiet times always, and everywhere, characterized by smaller fractal scaling measures than active times?

Affirmative answers would suggest the possibility that changes in statistics might then be used as local indicators of magnetospheric conditions, which may be useful to develop reliable warning and forecasting systems using information not available in geomagnetic indices.

A second objective is to determine the long-term fractal nature of the geomagnetic field at a local observation site. If the time series can be described as a particular statistical process - fractional Brownian motion for example - then this knowledge can be used for future space weather modeling purposes. Or scaling may be similar to that of multifractional Brownian motion (mBm) (Peltier and Lévy Vehel, 1995; Muniandy and Lim, 2001), where the scaling features depend on time. The statistical structure of the magnetometer time series provides key requirements to guide development of mathematical models. Magnetohydrodynamic models, for example, should correctly reproduce the correct statistical structure seen in the data.

In the following pages we present our examination of these questions. Section 2 describes the analysis techniques employed and important background to statistical methods. In Sect. 3 we describe the criteria used to select and process the data. Section 4 presents results. Finally, in Sect. 5 we form conclusions.

\section{Analysis techniques}

\subsection{Fractional Brownian motions}

A signal that displays fractional Brownian motion (fBm) can be expressed as (Mandelbrot and Van Ness, 1968) a stochastic integral of time integrations of fractional Gaussian noise:

$$
\begin{gathered}
B_{H}(t)=\frac{1}{\Gamma(H+1 / 2)}\left\{\int_{-\infty}^{0}\left[(t-s)^{H-1 / 2}-(-s)^{H-1 / 2}\right]\right. \\
\left.\mathrm{d} W(s)+\int_{0}^{t}(t-s)^{H-1 / 2} \mathrm{~d} W(s)\right\} .
\end{gathered}
$$

Here $W$ is a white noise process defined on $(-\infty, \infty)$, and $H \in(0,1)$ is known as the Hurst parameter. The Hurst exponent for the signal is its roughness averaged over many length scales. The covariance function is given by

$\operatorname{cov}\left\{B_{H}(s), B_{H}(t)\right\}=\frac{1}{2}\left\{|s|^{2 H}+|t|^{2 H}-|s-t|^{2 H}\right\}$,

so that $B_{H}(0) \equiv 0$ and $\operatorname{var}\left\{B_{H}(t)\right\}=t^{2 H}$. This means that for the special case $H=1 / 2$, fBm reduces to the wellknown random walk. Typically, fBm is nonstationary, and thus detection of the presence of memory is a delicate task. It has been observed in a variety fields, including hydrology 
(Neuman and Federico, 2003), geophysics (Wanliss and Antoine, 1995; Frisch, 1997), biology (Collins and De Luca, 1994), telecommunication networks (Taqqu et al., 1997), and others.

Traditional estimates of possible fractal scaling employ power spectral density (PSD) analysis. The slope $\beta$ obtained from the plot of PSD $P(v)$ vs. frequency $v$ determines the correlation level of the signal. If $P(v) \propto v^{-\beta}$, and $-1<\beta<$ 1 then the signal is fractional Gaussian noise (fGn) and it is stationary, which means that the signal is statistically invariant by translation in time. When the signal $B(t)$ displays fractional Brownian motion ( $\mathrm{fBm})$ both the real and imaginary components of the Fourier amplitudes are Gaussiandistributed random variables (Hergarten, 2002). As well, the mean of the Fourier amplitudes can be given by $\overline{\varphi(v)}=0$ and $\varphi(v) \varphi(v)^{*}=P(v) \delta\left(v-v^{\prime}\right)$. The last term is the Dirac delta function.

This means that for $\beta=2, \mathrm{fBm}$ reduces to the random walk with power law spectrum varying as an inverse square. Signals with scaling exponents above $\beta=2$ are called persistent, because if the data at some point have $B\left(t_{i+1}\right)>$ $B\left(t_{i}\right)$, for example, then the probability is greater than 0.5 that $B\left(t_{i+2}\right)>B\left(t_{i+1}\right)$. Signals with exponents below 2 are called antipersistent because if $B\left(t_{i+1}\right)>B\left(t_{i}\right)$, the probability is greater than 0.5 so that $B\left(t_{i+2}\right)<B\left(t_{i+1}\right)$.

If the signal is $\mathrm{fBm}$, it exhibits power-law scaling with slope $1<\beta<3$. In this case the signal is nonstationary but has stationary increments over a range of scales. For fBm

$\beta=2 H+1$,

where $H$ is the scaling exponent, also known as the Hurst exponent. Brownian motion marks the special case where $\beta=2(H=0.5)$.

Typically, fBm is nonstationary, and detection of memory is a delicate task. Nonstationarity means that statistical properties are not constant through the signal, and one cannot use traditional analysis methods that assume stationarity. The problem with spectral analysis, certainly in the context of the present study, is that it assumes a stationary signal. When implemented with nonstationary signals, spectral analysis is incapable of distinguishing frequency content hidden by the presence of $n$ th-order polynomial trends and requires long time series for reasonable accuracy (Stanley et al., 1999).

\subsection{Detrended fluctuation analysis}

Although much effort is focused on developing reliable estimators of long-range correlations, there appears to be no clear community consensus on the methods best suited for various types of data. In addition, tests of synthetic time series show that the performance of estimators varies when using different generators of long-range dependent data (Shao et al., 2012).

To determine the self-similarity parameter $H$ we implement detrended fluctuation analysis (DFA) developed by
Peng et al. (1995) and recently implemented in space physics research by Wanliss $(2004,2005)$, and subsequent papers. The technique determines the scaling exponent of nonstationary signals and provides better precision than the power spectral analysis and other more modern techniques. DFA is a modified root mean squared analysis of a random walk designed specifically to be able to deal with nonstationarities in nonlinear data, and is among the most robust of statistical techniques designed to detect long-range correlations in time series (Taqqu et al., 1995; Cannon et al., 1997; Blok, 2000; Shao et al., 2012). DFA has been shown to be robust to the presence of trends (Hu et al., 2001) and nonstationary time series (Kantelhardt et al., 2002; Chen et al., 2002). Bryce and Sprague (2012) argue that DFA has significant problems but they consider only the limited case of fGns with $H=0.3$, generated using the Davies-Harte algorithm. Shao et al. (2012) consider these and other recent criticisms of DFA and conclude that DFA "remains the method of choice" when the trend in the data is not a priori known.

Briefly, the DFA methodology begins by removing the mean, $\bar{B}$, from the time series, $B(t)$, and then integrating

$y(k)=\sum_{t=1}^{k}[B(t)-\bar{B}]$.

The new time-series is then divided into boxes of equal length, $n$ (Fig. 2). The trend, represented by a least-squares fit to the data, is removed from each box; the trend is typically a linear, quadratic, or cubic function. Box $n$ has its abscissa denoted by $y_{n}(k)$. Next the trend is removed from the integrated time series, $y(k)$, by subtracting the local trend, $y_{n}(k)$, in each box.

For a given box size $n$, the characteristic size of the fluctuations, denoted by $F(n)$, is then calculated as the root mean squared deviation between $y(k)$ and its trend in each box

$F(n)=\sqrt{\frac{1}{N} \sum_{k=1}^{N}\left[y(k)-y_{n}(k)\right]^{2}}$.

The presence of scaling is indicated by a power-law relationship between $F(n)$ and $n$ as follows:

$F(n) \propto n^{\alpha}$,

where $\alpha=H(0<\alpha<1)$ is the scaling exponent. The slope of a $\log -\log$ curve of $F(n)$ vs. $n$ indicates the value of the scaling exponent. For our data $n$ is in units of s, and $F(n)$ in units of $\mathrm{nT}^{2}$. If $\alpha=0.5$ then the signal is white noise. A value of $\alpha<0.5$ indicates uncorrelated data (antipersistent) and if $0.5<\alpha<1$ then there is correlation in the time series (i.e., long-term memory).

\section{Data selection}

In order to characterize the latitudinal variation of magnetosphere fractal scaling, we use the 190,210 , and $250^{\circ}$ 


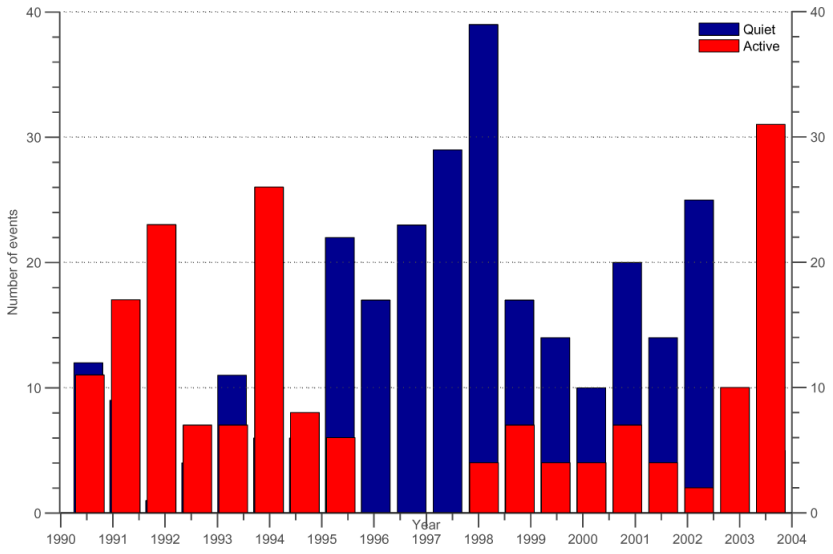

Fig. 1. Histogram of active (red) and quiet (blue) events.

magnetic meridian data (Yumoto et al., 1996). These data feature near-continuous, high-resolution (1 s cadence) data from a magnetometer chain spanning almost $160^{\circ}$ in geomagnetic latitude. Magnetometer stations are all close to $210^{\circ}$ geomagnetic longitude. Tables $1-3$ provide the station locations, given in geographic, magnetic, and $L$-shell coordinates.

We chose the 3-hour Kp index to discriminate between different levels of magnetospheric activity. We might have adopted other indices, for example DST or AE, but we chose $\mathrm{Kp}$ since we considered that it, as a mid- to high-latitude index, would best reflect the mean magnetospheric activity over the wide range of latitudes for the magnetometer data.

Several methods to classify geomagnetic activity using the Kp index have been proposed and used by different authors. Bartels (1963) used $\mathrm{Kp} \leq 1$ as an indicator of quiet periods, and $\mathrm{Kp} \geq 4$ as an indicator of disturbed periods (Rangarajan and Iyemori, 1997). In this work our interest focuses on two averaged geomagnetic states: active and quiet, so we adopted the Bartels criteria.

Data selected for quiet times (QT) are based on those $24 \mathrm{~h}$ periods between 1990 and 2003 where $\mathrm{Kp} \leq 1$. Active events (AT) were selected from $24 \mathrm{~h}$ periods of time having $\mathrm{Kp} \geq 4$. No overlap of events was allowed. In other words, if for instance $\mathrm{Kp} \geq 4$ for 48 consecutive hours, then there are two AT events, one beginning at the start of the interval, the other after the elapse of $24 \mathrm{~h}$. But if $\mathrm{Kp} \geq 4$ for $40 \mathrm{~h}$ we consider this as only one AT event, comprising the first $24 \mathrm{~h}$. Using non-overlapping events helps prevent any single period dominating and biasing the overall statistics.

With these criteria we find a number of 178 active day and 287 quiet day events. Figure 1 shows the yearly distribution of all the events selected, both active and quiet. Most of the active events (red) are close to solar maxima, while most of the quiet events congregate near solar minimum (around 1997).

Local time variations are known to exist in geomagnetic fluctuations, for instance the solar quiet fluctuations near noon (Wanliss and Showalter, 2006) which are absent at local
Table 1. Location of $190^{\circ}$ magnetometer stations used in this study.

\begin{tabular}{lcrrrrr}
\hline \multirow{2}{*}{ Station } & IAGA & \multicolumn{2}{c}{ Geographic } & \multicolumn{2}{c}{ CGM } & L Val \\
& code & Lat & Long & Lat & Long & \\
\hline Tixie & TIK & 71.6 & 129.0 & 65.7 & 197.1 & 5.89 \\
Lunping & LNP & 25.0 & 121.2 & 13.8 & 189.5 & 1.06 \\
Muntinlupa & MUT & 14.4 & 121.0 & 3.6 & 191.6 & 1.00 \\
Pontianak & PTN & -0.1 & 109.2 & -11.4 & 180.5 & 1.04 \\
Learmonth & LMT & -22.2 & 114.1 & -34.1 & 185.0 & 1.46 \\
Katanning & KAT & -33.7 & 117.6 & -46.6 & 188.2 & 2.12 \\
\hline
\end{tabular}

Table 2. Location of $210^{\circ}$ magnetometer stations.

\begin{tabular}{lcrrrrr}
\hline \multirow{2}{*}{ Station } & IAGA & \multicolumn{2}{c}{ Geographic } & \multicolumn{2}{c}{ CGM } & L Val \\
& code & Lat & Long & Lat & Long & \\
\hline Kotel'nyy & KTN & 75.9 & 137.7 & 69.9 & 201.0 & 8.50 \\
Chokhurdakh & CHD & 70.6 & 147.9 & 64.7 & 212.1 & 5.46 \\
Zyryanka & ZYK & 65.7 & 150.8 & 59.6 & 216.7 & 3.91 \\
Magadan & MGD & 60.0 & 150.9 & 53.6 & 218.7 & 2.83 \\
St. Paratunka & PTK & 52.9 & 158.2 & 46.3 & 225.9 & 2.10 \\
Moshiri & MSR & 44.4 & 142.3 & 37.6 & 213.2 & 1.59 \\
Rikubetsu & RIK & 43.5 & 143.8 & 34.7 & 210.8 & 1.48 \\
Onagawa & ONW & 38.4 & 141.5 & 31.6 & 212.5 & 1.38 \\
Kagoshima & KAG & 31.5 & 130.7 & 25.1 & 202.2 & 1.22 \\
Yamakawa & YMK & 31.19 & 130.62 & 24.85 & 202.13 & 1.21 \\
Chichijima & CBI & 27.2 & 142.3 & 20.6 & 213.0 & 1.14 \\
Guam & GUA & 13.6 & 144.9 & 4.6 & 214.7 & 1.01 \\
Yap & YAP & 9.3 & 138.5 & -0.3 & 209.0 & 1.00 \\
Biak & BIK & -1.1 & 136.1 & -12.2 & 207.3 & 1.05 \\
Wewak & WEW & -3.6 & 143.6 & -14.4 & 215.3 & 1.06 \\
Darwin & DAW & -12.4 & 130.9 & -23.1 & 202.7 & 1.18 \\
Weipa & WEP & -12.7 & 141.9 & -23.0 & 214.3 & 1.18 \\
Birdsville & BSV & -25.8 & 139.3 & -36.9 & 213.1 & 1.56 \\
Dalby & DLB & -27.2 & 151.2 & -37.1 & 226.8 & 1.57 \\
Canberra & CAN & -35.3 & 149.0 & -46.0 & 226.1 & 2.07 \\
Adelaide & ADL & -34.7 & 138.7 & -46.5 & 213.7 & 2.11 \\
\hline
\end{tabular}

Table 3. Location of $250^{\circ}$ magnetometer stations.

\begin{tabular}{lcrrrrr}
\hline Station & IAGA & \multicolumn{2}{c}{ Geographic } & \multicolumn{2}{c}{ CGM } & L Val \\
& code & Lat & Long & Lat & Long & \\
\hline Kotzebue & KOT & 66.9 & 197.4 & 64.5 & 249.7 & 5.40 \\
Ewa Beach & EWA & 21.3 & 202.0 & 22.7 & 269.4 & 1.17 \\
Macquarie Isl. & MCQ & -54.5 & 158.9 & -64.5 & 247.8 & 5.40 \\
\hline
\end{tabular}

midnight. The extent to which such variations influence fractal scaling is unknown. It is partly because of this unknown variability that we use a $24 \mathrm{~h}$ selection criterion. In this case the station samples every local time, reducing the likelihood of unknown local time variability dominating the statistical analysis. In essence, a $24 \mathrm{~h}$ period is needed since this allows enough time for any particular magnetometer to sample each different longitudinal region of the magnetosphere. Our analysis thus uses data in a way that is analogous to geomagnetic index data. Geomagnetic indices convolve data from many magnetometers, measured at the same universal time, but at many different local times (and latitudes). Our analysis takes a station at a single geographic location and allows it to sweep through every local time. Thus the possibility that fractal scaling also depends on longitude is negated since the entire longitudinal range is sampled. In addition, this period 
allows enough data so that random fluctuations are averaged out.

Studies that use geomagnetic indices implicitly include local time effects due to the nature of the convolution of data to form the index. This produces pitfalls that are usually ignored in the numerous studies of index data. These difficulties do not negate the statistical analyses of index data, as is evident from voluminous publications. Similarly, and though we offer no solution to this issue, our analysis adds to our body of knowledge.

Since not all of the magnetometer stations are operational at the same time, we scanned through the 190,210, and $250 \mathrm{~mm}$ data, selecting data from stations when available, that overlap with the Kp selection criteria.

In the end we used data from 40 magnetometers across almost $160^{\circ}$ magnetic latitude. Since the magnetometer data are often noisy, subject to DC shifts, spikes, monochromatic constant amplitude oscillations, and other spurious effects, it is necessary to further cull the magnetometer data. Because of these difficulties with quality we carefully examine magnetometer data by eye to eliminate spurious data. We instituted a laborious routine whereby data were visually examined before analysis. From the Kp data intervals of $24 \mathrm{~h}$ were selected which satisfied the appropriate quiet and active criteria. Next, we visually inspected all of the $210 \mathrm{~mm}$ data in $24 \mathrm{~h}$ blocks falling in the Kp selected intervals. Data having spurious features, mentioned above, were eliminated. Once this was done we still found good quality data for a total of $2.6 \times 10^{8}$ QT data points and $1.2 \times 10^{8}$ AT data points.

Figure 2 gives a sample distribution function of the magnetic field for QTs and ATs. In this plot the solid curve characterizes the $X$ component magnetic field behavior during AT, while the dashed curve represents the QT measured at the MSR station.

\section{Results}

Once data are selected we apply DFA. Because we analyze $24 \mathrm{~h}$ intervals there are $N=86400$ data points in each series. We use a box size beginning at $8 \mathrm{~s}$ and terminating at $N / 4=21600$ s. We use such a cutoff since the low and high box-number edges should be treated with caution. The first few points at the low end must be disregarded since detrending here removes too much of the fluctuation. For larger values of the box size, there are too few boxes for a proper average to be made - a finite size effect - hence we disregard those values. Selecting a box size terminating at less than $N / 4$ still allows reasonable estimates of scaling exponents. However, following $\mathrm{Hu}$ et al. (2001) and Kantelhardt et al. (2002) we opt for the larger box size since it allows more reliable estimates of the scaling exponent over more than two decades of magnitude.

Figure 3 shows an example of the fluctuation function $F(n)$ against scale size $n$ for the quiet event of 20 January

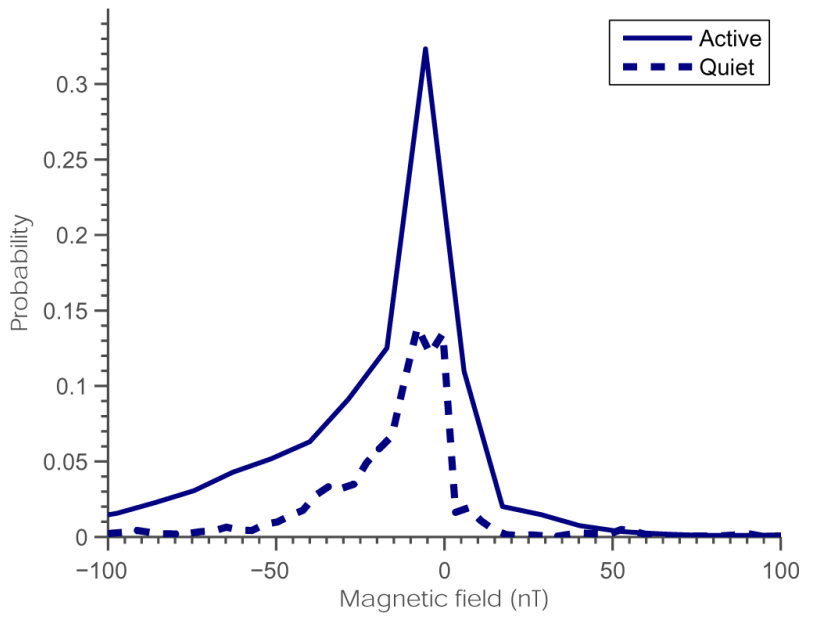

Fig. 2. Distribution functions for the active and quiet events analyzed for the $X$ component magnetic field at the MSR magnetometer station. The dashed line represents the averaged distributions for the quiet events and the solid line represents the averaged distributions for the active events.

2000, for three geomagnetic components. The inset shows the magnetic field components for the Biak magnetometer station (BIK). For this event scaling is present for almost four decades of time, with $F(n)$ shown by the circles. Some deviation from the best fit (solid line) is clear near the smallest and largest scales, and is common for all events. Accordingly, to analyze these data we use least-squares fitting over a range from $100 \mathrm{~s}$ to $12 \mathrm{~h}$. For this event the scaling exponent is about $\alpha \sim 0.8-0.9$. The fits are excellent and uncertainties are on the order of $1-2 \%$ of the value for the scaling exponent. We find that smaller box sizes yield similar scaling exponents, but based on a linear fitting on a smaller scale range, resulting in larger uncertainty.

Figures 4 and 5 summarize the results obtained for the distribution of scaling exponent $\alpha$ for the 3027 quiet and 1422 active events computed for all latitudes. The figures show a Gaussian best fit. Other distributions might be fitted, such as Rayleigh, Maxwell, normal, and log-normal distributions (Wanliss and Showalter, 2006). For quiet events the statistics are fit best by a Gaussian. Active data appear slightly different, having the inkling of stable, heavy-tailed distributions, mainly in the 4-component results. Table 4 summarizes these results, showing that the standard deviations are quite large. The mean values of the scaling exponents $\alpha$ are statistically similar, with $\alpha \sim 0.65-0.7$ irrespective of quiet or active times.

As found previously, by Wanliss and Reynolds (2003) and Yu et al. (2010), we find a broad range of scaling exponents, as evidenced by large standard deviations from the mean. This is what one might expect of exponents calculated from magnetometers spanning magnetic latitudes from the equator to auroral regions; if fractal scaling is latitude dependent, a broad $\alpha$ distribution must be the consequence. 

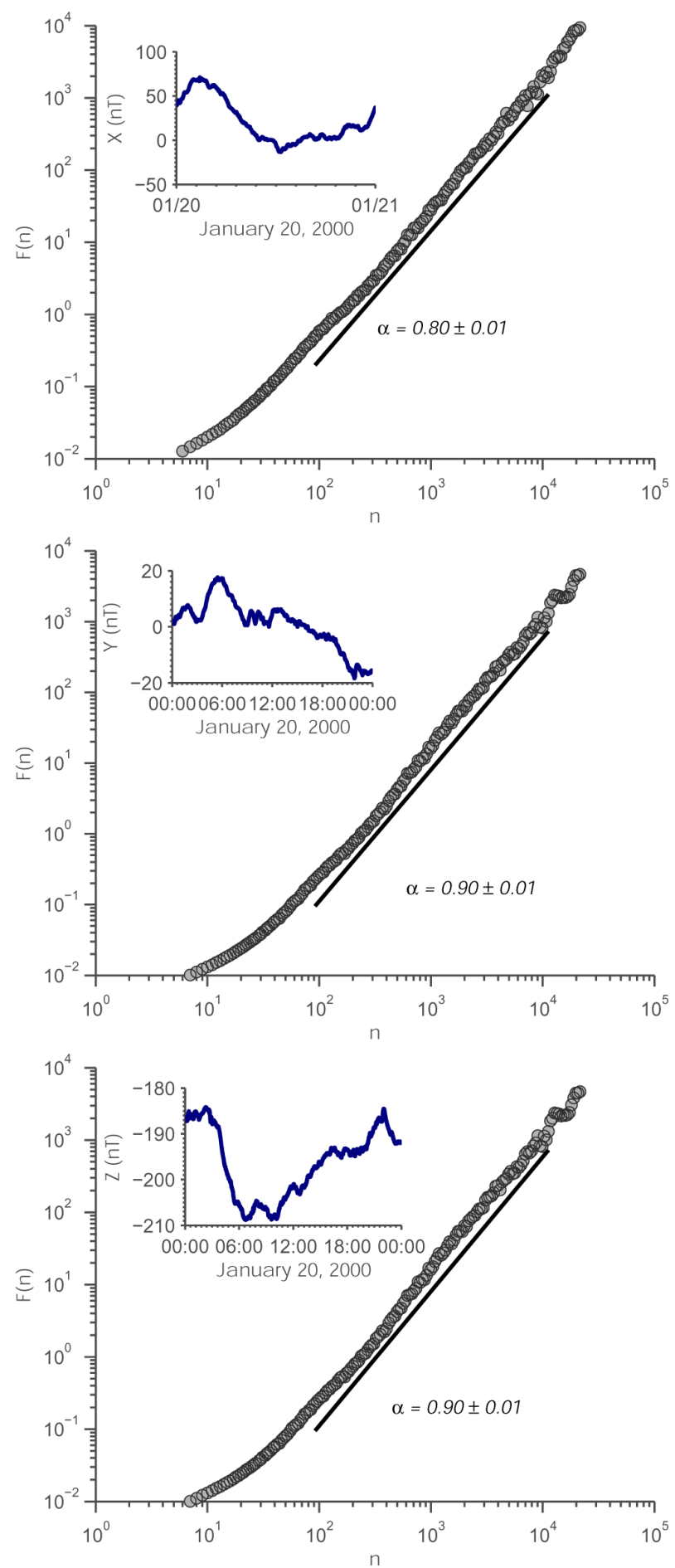

Fig. 3. Circles show an example of the fluctuation versus box size for the quiet event beginning on 20 January 2000. Solid curve below the circles is the best fit linear curve to the data (shifted) and slope $\alpha$ is shown on each graph. The inset in this example shows the component magnetometer data from the BIK station used to compute the fluctuation curves.
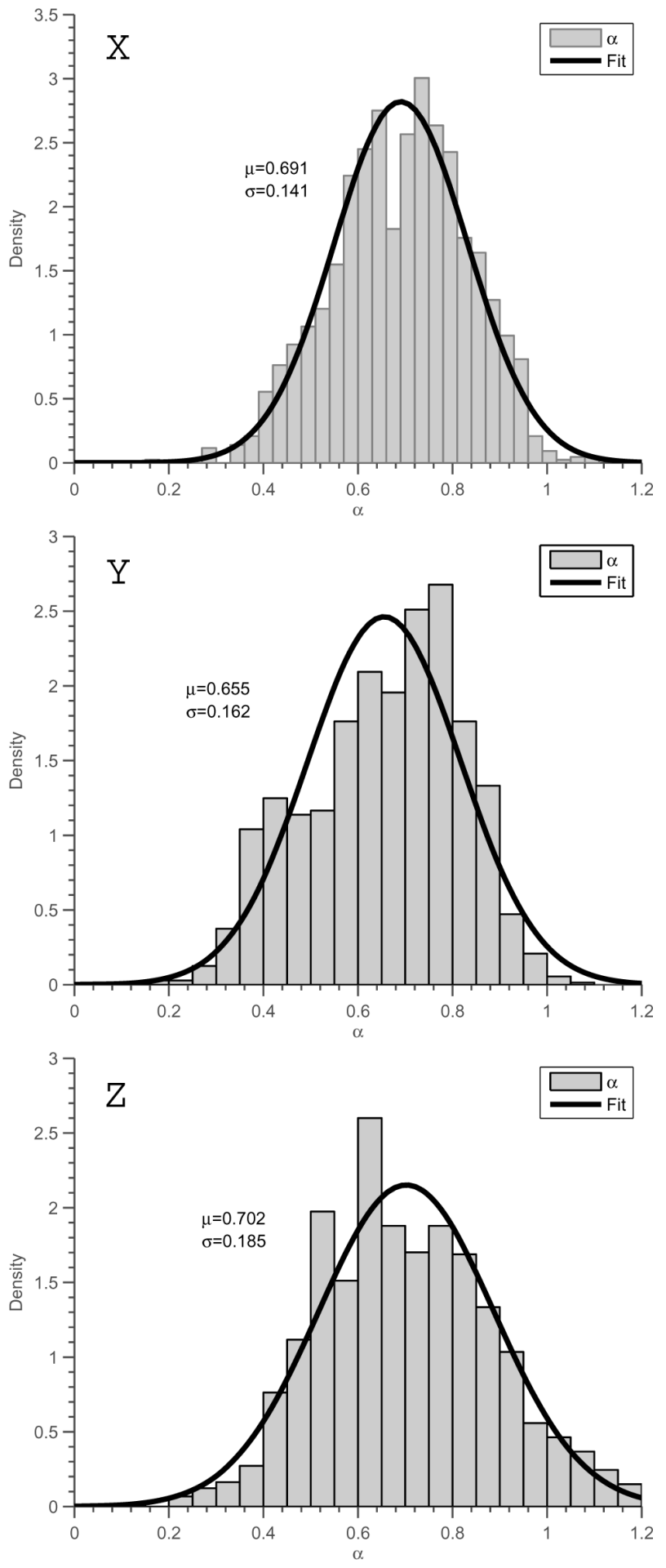

Fig. 4. Distributions of the fractal scaling exponent for quiet events, for all latitudes. These data are fitted to a normal distribution (solid black), with the mean and standard deviation given in the figures.

We find that when all latitudes are included in the analysis there is no statistical difference between fractal exponents measured during quiet and active intervals. However, 
Table 4. Scaling exponents for the quiet and active events computed for all latitudes.

\begin{tabular}{lccc}
\hline & $X \alpha$ & $Y \alpha$ & $Z \alpha$ \\
\hline Quiet & $0.64 \pm 0.13$ & $0.65 \pm 0.14$ & $0.67 \pm 0.16$ \\
Active & $0.69 \pm 0.14$ & $0.66 \pm 0.16$ & $0.70 \pm 0.19$ \\
\hline
\end{tabular}

there is some difference in the overall distribution functions, and the distribution of active component $Y$ component data is slightly asymmetric with a heavier tail for $\alpha<0.5$.

Skewness is a measure of asymmetry of the data around the sample mean. It is positive(negative) when the data spread out more to the right(left). For a normal distribution, skewness is zero. We find the skewness for quiet events for $X, Y$, and $Z$ magnetic field components to be, respectively $[-0.40,-0.16,-0.09]$. For active events the corresponding skewness is $[-0.20,-0.40,0.26]$. None of these distributions are significantly different from a normal distribution for the means are essentially the same as the medians.

Next we examine whether we can distinguish the latitudinal dependency from the rest of the variability. We attempt this by considering the average of many scaling exponents that were computed at a single station, calculating the mean and standard deviation of them all. Figure 6 shows the average scaling exponent against geomagnetic latitude for quiet (magenta circles) and active (red triangles) events. The bestfit polynomial shown is a quadratic function. We fitted various polynomials and found that the second-order polynomial gave the best fit for $X$ and $Y$. Our preference was to fit the simplest possible model that provides reasonable fits. The order of the best-fit polynomial is determined on the basis of (1) visual examination of the residuals, (2) goodness-offit statistics, and (3) confidence bounds. The best-fit is computed with the Levenberg-Marquardt algorithm for nonlinear least squares (Seber and Wild, 2005).

A latitudinal variability emerges from these data. For $X$ and $Y$ geomagnetic components there is a tendency for the scaling exponent to increase as the magnetometer approaches the geomagnetic equator. No such variation is plain in $Z$. In addition this behavior is consistent across the quiet to active events, thus independent of geomagnetic activity. As well, for each magnetic component the best-fit active events tend to have scaling exponents larger than for quiet times.

To explore whether these quiet and active event fractal scaling exponents are significantly different from the null hypothesis - that the difference is due purely to randomness we applied the Students paired $t$ test to the distributions. The important output of the paired $t$ test is the value of $p$, which is the probability the difference in the means of the two distributions is due to random variation. The test focuses on the difference between the paired data and reports the probability that the actual mean difference is consistent with zero.

We found for $[X, Y, Z]$ that $p=[0.01,0.89,0.23]$. This shows that statistical differences between the sets are in-
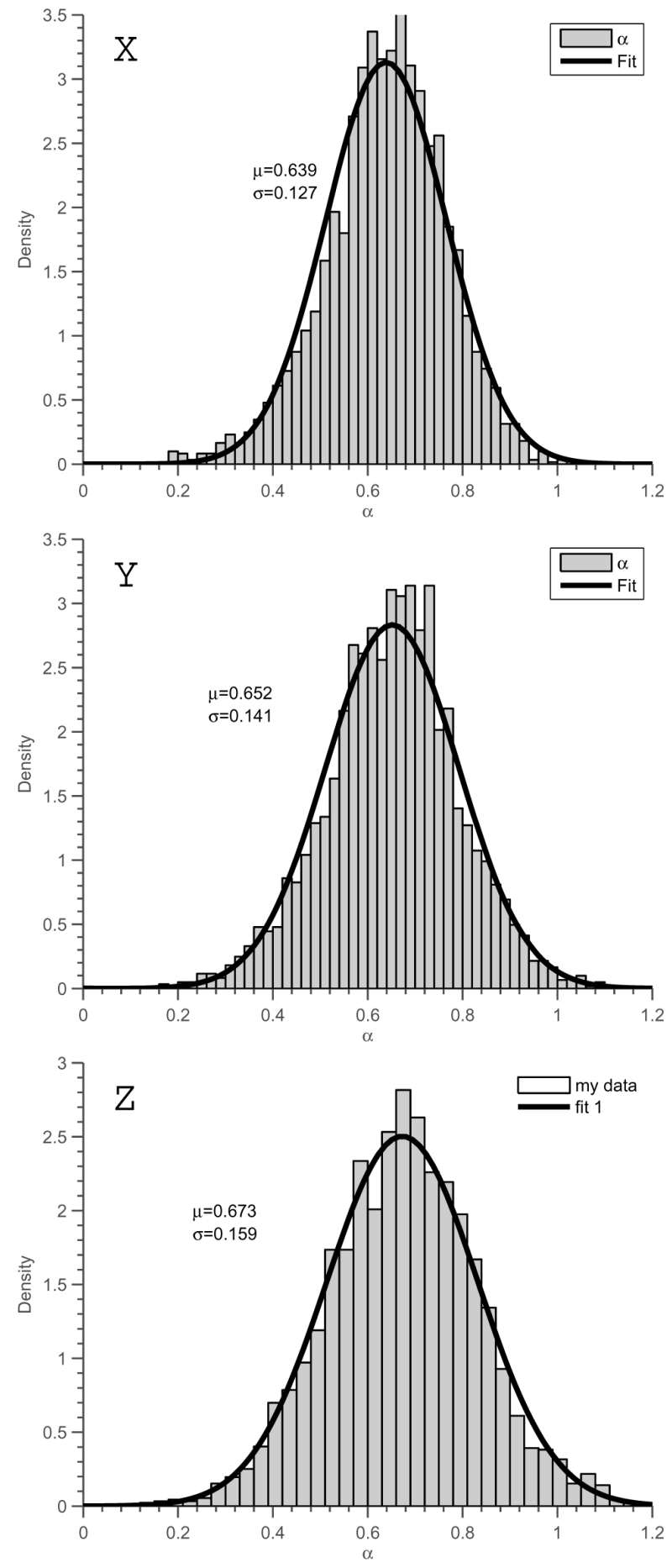

Fig. 5. Distributions of the fractal scaling exponent for active events, for all latitudes. These data are fitted to a normal distribution (solid black), with the mean and standard deviation given in the figures.

significant for the $Y$ and $Z$ components, particularly $B Y$. Though their fluctuations are very different, the overall nonlinear statistics across quiet and active intervals are statistically indistinguishable. However, for $X$ the statistical 

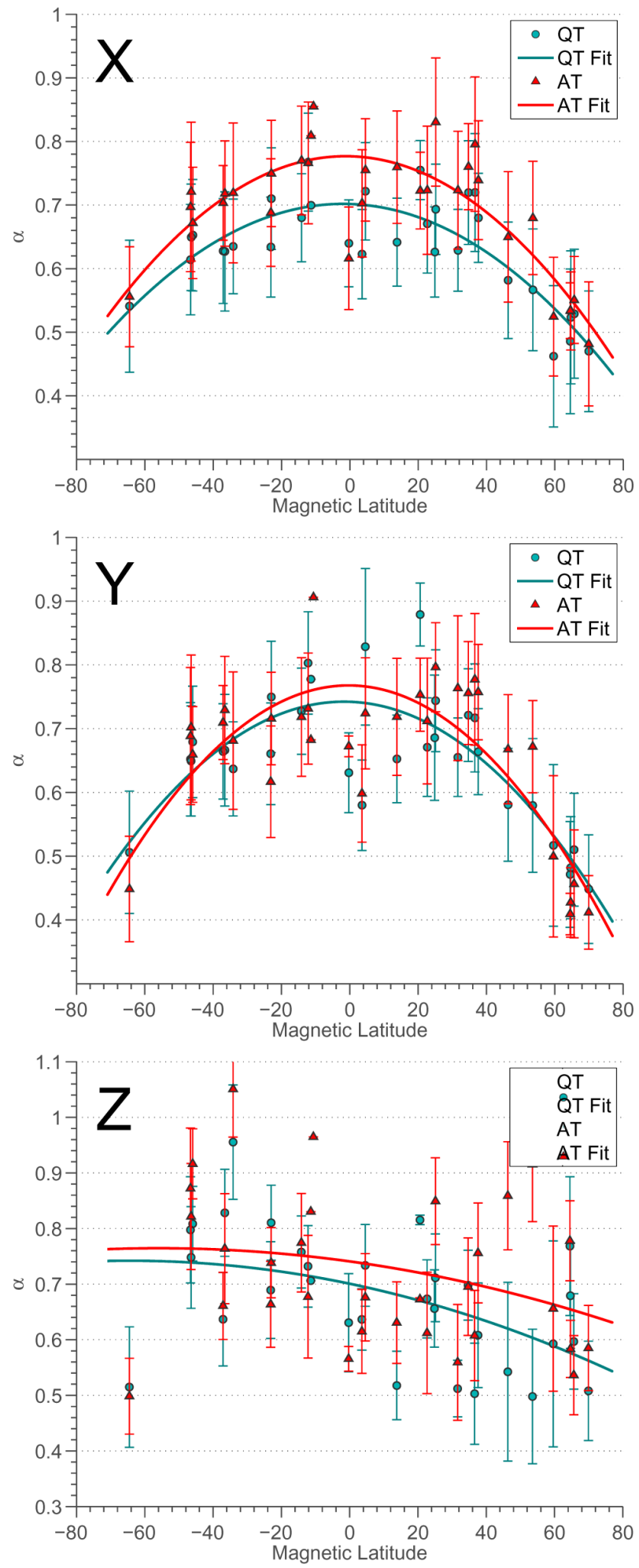

Fig. 6. Fractal scaling exponent for quiet (magenta circles) and active (red triangles) events as a function of latitude for different components of the magnetic field. The best-fit polynomials are shown in red (active) and magenta (quiet). differences are significantly different from zero - active events have significantly larger scaling exponents than for quiet events.

\section{Conclusions}

In this paper we characterized fractal behavior of the magnetic field time series obtained from ground-based observatories across a wide range of latitudes. We used the 190, 210 , and $250^{\circ}$ magnetic meridian data. We classified these data into quiet (QT) and active (AT) periods using the Kp in$\mathrm{dex}$ as the discriminator. For quiet intervals, we required $\mathrm{Kp} \leq 1$ for $24 \mathrm{~h}$. Similarly, qualifying as an active interval required $\mathrm{Kp} \geq 4$ for $24 \mathrm{~h}$.

We use the DFA technique to compute fractal scaling exponents due to its performance in dealing with nonstationary data. We found evidence that fractional Brownian motion is ubiquitous across all the latitudes encompassed by this study (see e.g., Fig. 2).

Differences presented between QT and AT are less clear for $Y$ and $Z$, but significant for $X$ (Fig. 6), with events tending to have larger scaling exponents. This implies that active events possess more of a memory effect, while quiet events have data tending to be more random. Scaling exponents at individual stations are variable, irrespective of magnetosphere activity organized in terms of Kp. The latter result suggests that a suitable model of geomagnetic fluctuations is not monofractal but multifractional, i.e., the scaling exponent changes as a function of time. The distributions for quiet and active events encompasses marked differences as the result of different processes dominating the dynamics of the magnetosphere. During quiet times energy is stored and slowly burned, keeping the magnetosphere in a relative low-energy state. But during active times higher energy influx from the solar wind causes the magnetosphere to move to higher energy states, where strong nonlinear processes can dominate the dynamical release of energy.

Both the $X$ and $Y$ geomagnetic components show significant and correlated latitudinal variation, with scaling exponents increasing as the geomagnetic equator is approached. $Z$ shows no such correlation, suggesting that for $Z$ the fingerprints of local magnetic activity are not conserved during the timescale of a particular quiet or active event as determined by our selection criteria.

Our results thus suggest that the magnetic field at a single geomagnetic latitude is better described as a multifractional Brownian motion $(\mathrm{mfBm})$ rather than as a $\mathrm{fBm}$ process. The $\mathrm{mfBm}$ is a generalized version of $\mathrm{fBm}$ in which the scaling exponent $\alpha$ is no longer a constant, but a function of the time index (Peltier and Lévy Vehel, 1995). In this case the increments of $\mathrm{mfBm}$ are nonstationary and the process is no longer self-similar.

Another possibility, not explored in this work, is that the well-defined scaling properties of our data are the result of a 
multifractal process. That is to say, there is no unique scaling exponent, but the presence of a full hierarchy of them (possibly with a stationary distribution, although not required in the generalized scale invariance framework). For instance, Bianchi and Planese (2007) found that in some cases the partition function as well as the scaling function of the $\mathrm{mBm}$, i.e., of a generally non-multifractal process, behave as those of a genuine multifractal process. Indeed, previous work by Wanliss et al. (2005) and Yu et al. (2010) demonstrate the presence of multifractality in magnetospheric data at higher latitudes.

Although this work has only considered the hypothesis of multifractionality, it appears from a synthesis of multiple papers, that the nature of the geomagnetic disturbances (magnetospheric response to solar wind changes) is composed of the two different processes, multifractality (dependence of the increment statistics on the timescale) and multifractionality (corresponding to a unique (across scales), but nonstationary, scaling exponent). The former depends strongly on the solar wind variability and turbulent nature, and the latter is mainly due to the internal magnetospheric dynamics (with a special emphasis to the plasma and current sheet conditions), which could imply a different Markovian nature of the geomagnetic response at certain timescales. The different character between multifractality and multifractionality should manifest in a scale, independent of the fluctuations (increment) statistics. We leave these more detailed explorations to a later work.

Our results can serve as a guide, suggesting the required statistical structure for mathematical models of magnetospheric activity. We also offer a possible explanation relating the physics of QT and AT with their different fractal exponents. Our results are consistent with Consolini and Lui (2000), who examined scaling properties of magnetic fluctuations in the magnetotail. They consistently found a lower scaling exponent before current disruption, followed by higher values afterward. They interpreted the change in scaling exponent as a reorganization during current disruption. Since our ground-based data map the magnetosphere along magnetic field lines, the differences suggest the possibility that, at least for $X$, the fractal statistics offer another way to monitor transitions from QT to AT.

In conclusion, we have shown evidence that, for ground magnetometer data, average fractal scaling does vary in a consistent way as a function of latitude. And finally, at least for the $X$ component, magnetospheric quiet times are characterized always, and everywhere, by smaller fractal scaling measures than active times.

Acknowledgements. This material is based on work supported by the National Science Foundation under Grant No. 1104364.

Edited by: D. Schertzer

Reviewed by: A. S. Sharma and one anonymous referee

\section{References}

Anh, V., Yu, Z.-G., and Wanliss, J. A.: Analysis of global geomagnetic variability, Nonlin. Processes Geophys., 14, 701-708, doi:10.5194/npg-14-701-2007, 2007.

Balasis, G., Daglis, I. A., Papadimitriou, C., Anastasiadis, A., Sandberg, I., and Eftaxias, K.: Quantifying Dynamical Complexity of Magnetic Storms and Solar Flares via Nonextensive Tsallis Entropy, Entropy, 13, 1865-1881, 2011.

Bartels J.: Discussion of time variations of geomagnetic indices Kp and Ap, Ann. Geophys., 19, 19323-19361, 1963.

Bartels, J., Heck, N. H., and Johnston, H. F.: The three-hour-range index measuring geomagnetic activity, J. Geophys. Res., 44, 411-454, 1939.

Bianchi, A. and Planese, A.: Modelling stock price movements: multifractality or multifractionality?, Quant. Financ., 7, 301319, 2007.

Bryce, R. and Sprague, K.: Forecasting conflict intensity: Afghanistan, Journal of Battlefield Technology, 15, 23-29, 2012.

Chen, Z., Ivanov, P. C., Hu, K., and Stanley, H. E.: Effect of nonstationarities on detrended fluctuation analysis, Phys. Rev. E, 65, 041107, doi:10.1103/PhysRevE.65.041107, 2002.

Collins, J. J. and De Luca, C. J.: Upright, correlated random walks: A statistical-biomechanics approach to human postural control system, Chaos, 5, 57-63, 1994.

Consolini, G. and De Michelis, P.: Non-Gaussian distribution functions of AE-index fluctuations: Evidence for time intermittency, Geophys. Res. Lett., 25, 4087-4090, 1998.

Consolini, G. and Lui, A. T. Y.: Symmetry breaking and nonlinear wave-wave interaction in current disruption: Possible evidence for a phase transition, in: Magnetospheric Current Systems, edited by: Ohtani S.-I., Fujii, R., Hesse, M., and Lysak, R. L., Geophys. Monogr. Ser., 118, p. 395, 2000.

Consolini, G., Marcucci, M. F., and Candidi, M.: Multifractal Structure of Auroral Electrojet Index Data, Phys. Rev. Lett., 76, 40824085, 1996.

Dobias, P. and Wanliss, J. A.: Intermittency of storms and substorms: is it related to the critical behaviour?, Ann. Geophys., 27, 2011-2018, doi:10.5194/angeo-27-2011-2009, 2009.

Frisch, U.: Turbulence, Cambridge University Press, New York, 1997.

Hergarten, S.: Self-Organized Criticality in Earth Systems, Springer, Berlin, Heidelberg, New York, 2002.

Hori, T., Lui, A. T. Y., Ohtani, S., C:son Brandt, P., Mauk, B. H., McEntire, R. W., Maezawa, K., Mukai, T., Kasaba, Y., and Hayakawa, H.: Storm-time convection electric field in the near-Earth plasma sheet, J. Geophys. Res., 110, A04213, doi:10.1029/2004JA010449, 2005.

Hu, K., Ivanov, P. C., Chen, Z., Carpena, P., and Stanley, H. E.: Effect of trends on detrended fluctuation analysis, Phys. Rev. E, 64, 011114, doi:10.1103/PhysRevE.64.011114, 2001.

Kantelhardt, J. W., Zschiegner, S. A., Koscielny-Bunde, E., Havlin, S., Bunde, A., and Stanley, H. E.:, Multifractal detrended fluctuation analysis of nonstationary time series, Physica A, 316, 87114, 2002.

Korth, A., Friedel, R. H. W., Henderson, M. G., Frutos-Alfaro, F., and Mouikis, C. G.: $\mathrm{O}^{+}$Transport into the ring current: Storm versus substorms, in: Disturbances in Geospace: The StormSubstorm Relationship, edited by: Sharma, A. S., Kamide, Y., 
and Lakhina, G. S., AGU, Washington, DC, Geoph. Monog. Series, 142, 59-73, 2003.

Mandelbrot, B. B. and Van Ness, J. W.: Fractional Brownian Motions, Fractional Noises and Applications, SIAM Rev., 10, 422437, 1968.

Muniandy, S. V. and Lim, S. C.: Modeling of locally selfsimilar processes using multifractional Brownian motion of Riemann-Liouville type, Phys. Rev. E, 63, 046104, doi:10.1103/PhysRevE.63.046104, 2001.

Neuman, S. P. and Federico, V. D.: Multifaceted Nature of Hydrogeologic Scaling and Its Interpretation, Rev. Geophys., 41, 1014, doi:10.1029./2003RG000130, 2003.

Peltier, R. and Lévy Vehel, J.: Multifractional Brownian motion: Definition and preliminary results, INRIA, Technical report No. 2645, 39 pp., 1995.

Peng, C. K., Havlin, S., Stanley, H. E., and Goldberger, A. L.: Quantification of scaling exponents and crossover phenomena in nonstationary heartbeat time series, CHAOS, 5, 82-87, 1995.

Pulkkinen, T. I., Partamies, N., Huttunen, K. E. J., Reeves, G. D., and Koskinen, H. E. J.: Differences in geomagnetic storms driven by magnetic clouds and ICME sheath regions, Geophys. Res. Lett., 34, L02105, doi:10.1029/2006GL027775, 2007.

Rangarajan, G. K. and Iyemori, T.: Time variations of geomagnetic activity indices Kp and Ap: an update, Ann. Geophys., 15, 12711290, doi:10.1007/s00585-997-1271-z, 1997.

Seber, G. A. F. and Wild, C. J.: Nonlinear Regression, John Wiley \& Sons, Inc., Hoboken, NJ, USA, doi:10.1002/0471725315, 2005.

Sharma, A. S. and Veeramani, T.: Extreme events and long-range correlations in space weather, Nonlin. Processes Geophys., 18, 719-725, doi:10.5194/npg-18-719-2011, 2011.

Stanley, H. E., Amaral, L. A. N., Goldberger, A. L., Havlin, S., Ivanov, P. Ch., and Peng, C. K.: Statistical physics and physiology: monofractal and multifractal approaches, Physica A, 270, 309-324, 1999.

Takalo, J., Timonen, J., Klimas, A., Valdivia, J., and Vassiliadis, D.: Nonlinear energy dissipation in a cellular automaton magnetotail field model, Geophys. Res. Lett., 26, 1813-1816, 1999.

Taqqu, M. S., Teverovsky, V., and Willinger, W.: Estimators for long-range dependence: An empirical study, Fractals, 3, 785$798,1995$.
Taqqu, M., Teverovsky, V., and Willinger, W.: Is network traffic selfsimilar or multifractal?, Fractals, 5, 63-73, 1997.

Vörös, Z.: On multifractality of high-latitude geomagnetic fluctuations, Ann. Geophys., 18, 1273-1282, doi:10.1007/s00585-0001273-6, 2000.

Wanliss, J.: Fractal properties of SYM-H during quiet and active times, J. Geophys. Res., 110, A03202, doi:10.1029/2004JA010544, 2005.

Wanliss, J. and Uritsky, V.: Understanding bursty behavior in midlatitude geomagnetic activity, J. Geophys. Res., 115, A03215, doi:10.1029/2009JA014642, 2010

Wanliss, J. A.: Nonlinear variability of SYM-H over two solar cycles, Earth Planets Space, 56, e13-e16, 2004.

Wanliss, J. A. and Antoine, L. A. G.: Geomagnetic micropulsations: Implications for high resolution aeromagnetic surveys, Explor Geophys., 26, 535-538, 1995.

Wanliss, J. A. and Dobias, P.: Space Storm as a Phase Transition, J. Atmos. Sol.-Terr. Phy., 69, 675-684, doi:10.1016/j.jastp.2007.01.001, 2007.

Wanliss, J. A. and Reynolds, M. A.: Measurement of the stochasticity of low-latitude geomagnetic temporal variations, Ann. Geophys., 21, 2025-2030, doi:10.5194/angeo-21-2025-2003, 2003.

Wanliss, J. A. and Showalter, K. M.: High-resolution global storm index: Dst versus SYM-H, J. Geophys. Res., 111, A02202, doi:10.1029/2005JA011034, 2006.

Wanliss, J. A., Anh, V. V., Yu, Z.-G., and Watson, S.: Multifractal modeling of magnetic storms via symbolic dynamics analysis, $\mathrm{J}$ Geophys. Res., 110, A08214, doi:10.1029/2004JA010996, 2005

Yu, Z.-G., Anh, V., Wang, Y., Mao, D., and Wanliss, J.: Modeling and simulation of the horizontal component of the geomagnetic field by fractional stochastic differential equations in conjunction with empirical mode decomposition, J. Geophys. Res., 115, A10219, doi:10.1029/2009JA015206, 2010

Yumoto, K. and the 210MM Magnetic Observation Group: The STEP 210 magnetic meridian network project, J. Geomagn. Geoelectr., 48, 1297-1310, 1996. 